\title{
Transparent boundary condition for beam propagation
}

\author{
G. Ronald Hadley \\ Sandia National Laboratories, Albuquerque, New Mexico 87185-5800
}

Received January 8, 1991

\begin{abstract}
A new boundary condition algorithm is presented that passes outgoing radiation freely with a minimum reflection coefficient (typically $10^{-5}$ ) while inhibiting the flux of incoming radiation. In contrast to the commonly used absorber method, this algorithm contains no adjustable parameters and is thus problem independent. It adapts naturally to a standard Crank-Nicholson difference scheme and is shown to be accurate and robust for both twoand three-dimensional problems.
\end{abstract}

The beam-propagation method is currently the most widely used tool for the investigation of complex optoelectronic structures such as tapered or bent waveguides and $Y$ junctions. ${ }^{1-3}$ Unfortunately this method is notoriously weak in modeling structures that permit radiation loss, since that radiation tends to reflect from the problem boundaries back into the solution region where it causes unwanted interference. The seriousness of this problem follows from the fact that virtually all the structures of interest do in fact result in scattered radiation. The most common way of preventing boundary reflection has been the insertion of artificial absorbing regions adjacent to the pertinent boundaries. 4 This procedure is accurate, provided that the absorbing region is carefully tailored, i.e., by using a small enough absorption gradient so that the absorber itself does not generate reflections and a thickness sufficient to absorb all radiation impinging upon the region. Unfortunately, ensuring that these conditions are properly met for each new problem is often a difficult and time-consuming process. Even when one is successful, the addition of extra problem zones results in computational penalties of run time and storage space. Recently, a different beam-propagation algorithm was reported (the socalled method of lines) that properly treats radiation loss through the problem boundaries. ${ }^{5}$ This method is quite effective for longitudinally uniform structures. Because it is essentially an eigenmode expansion technique, however, the treatment of problems containing longitudinally varying dielectric constants is hindered by the necessity of recalculating the eigenmode spectrum whenever the dielectric constant changes. Thus, this method is cumbersome at best for simulating a number of important structures such as tapered waveguides or $Y$ junctions.

In this paper I describe a new boundary condition algorithm that allows radiation to escape the problem freely without appreciable reflection while prohibiting the flux of radiation back into the problem region. This transparent boundary condition (TBC) employs no adjustable parameters and is thus problem independent. In addition, it is easily incorporated into a standard Crank-Nicholson differencing scheme and is applicable to longitudinally varying structures of interest for optoelectronics research.
A technical description of this new technique begins by considering the scalar paraxial beam-propagation equation. Since only the boundary region is of interest, we further restrict ourselves to the diffraction terms,

$$
\frac{\partial E}{\partial z}=\frac{i}{2 k} \frac{\partial^{2} E}{\partial x^{2}},
$$

where an additional $\exp (i k z)$ dependence was assumed in the derivation of Eq. (1). By simple manipulations, Eq. (1) may be rewritten in the useful energy conservation form

$$
\frac{\partial}{\partial z} \int_{a}^{b}|E|^{2} \mathrm{~d} x=\left.\frac{i}{2 k}\left(E^{*} \frac{\partial E}{\partial x}-E \frac{\partial E^{*}}{\partial x}\right)\right|_{a} ^{b} \equiv-F_{b}+F_{a},
$$

where $F_{b}$ represents the energy flux leaving the right boundary and $F_{a}$ represents that entering through the left boundary. Since the treatment of the two boundaries is essentially identical, we consider only the right boundary. We next make the important assumption that at this boundary $E=E_{0} \exp \left(i k_{x} x\right)$, where $E_{0}$ and $k_{x}$ are complex, and $k_{x}$ is for the moment unknown. With this assumption, $F_{b}$ becomes

$$
F_{b}=\frac{\mathcal{R}\left(k_{x}\right)|E(b)|^{2}}{k} \text {. }
$$

Therefore, as long as the real part of $k_{x}$ is positive, the contribution to the overall change in energy from this boundary will always be negative, i.e., radiative energy can only flow out of the problem region.

If we now consider the finite difference equivalent of Eq. (1) using a standard Crank-Nicholson scheme, it can be shown that the above energy balance relationship is preserved. Thus, assuming the same exponential dependence described above, we adjust the boundary value $E_{M}^{n}$ prior to the start of the $(n+1)$ th propagation step so that

$$
\frac{E_{M}^{n}}{E_{M-1}^{n}}=\frac{E_{M-1}^{n}}{E_{M-2}^{n}}=\exp \left(i k_{x} \Delta x\right) .
$$

This then determines $k_{x}$, and the boundary condition for the new propagation step is thus 


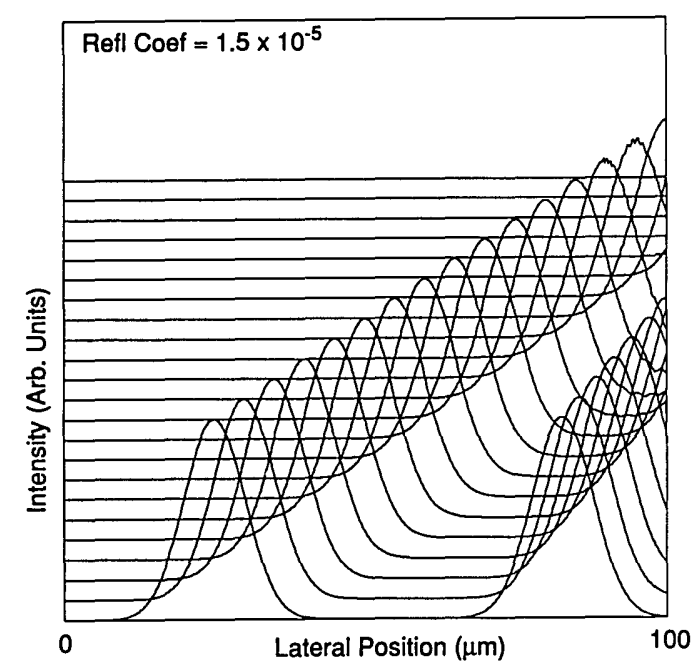

Fig. 1. Propagation of two Gaussian pulses through the transparent boundary at different angles. Shown are snapshots of the pulses at $30-\mu \mathrm{m}$ intervals.

$$
E_{M}^{n+1}=E_{M-1}^{n+1} \exp \left(i k_{x} \Delta x\right) .
$$

However, prior to the application of Eq. (5), the real part of $k_{x}$ must be restricted to be positive to ensure only radiation outflow.

An important feature of the above procedure is that $k_{x}$ is allowed to change as the problem progresses, thus eliminating the need for a problem-dependent adjustable parameter. However, the exact manner in which $k_{x}$ is determined is not an essential part of the method, so that the prescription given in Eq. (4) (which was used in obtaining the test results discussed below) should be looked on merely as a reasonable starting point.

The TBC procedure described above has been tested for accuracy and robustness on three test problems, the first two being two dimensional and the third being three dimensional. In each case the scalar paraxial propagation equation [the differenced version of Eq. (1) plus the extra dielectric function term] was solved with a standard tridiagonal algorithm. The three-dimensional simulation employed a split-step procedure (described in Ref. 6 as FDBPM), and the TBC algorithm was applied in a straightforward manner to each individual step.

The first test problem purports to provide a measure of the effective power reflectivity at the boundary by propagating two noninteracting Gaussian beams through the right boundary and determining the fraction of the initial power remaining in the solution region. The two beams are incident at angles of 5.7 and $11.5 \mathrm{deg}$, and their intensity profiles are shown in Fig. 1 at propagation distances spaced by $30 \mu \mathrm{m}$. These calculations employed 512 mesh points and a longitudinal step size of $0.2 \mu \mathrm{m}$. The beams had a free-space wavelength of $0.828 \mu \mathrm{m}$ and were propagated through a region that had a constant index of refraction of 3.3. As is vividly shown in Fig. 1, the waves virtually disappear into the boundary, which results in an effective reflection coefficient of $1.5 \times 10^{-5}$.

The second test problem involves calculation of the energy lost to radiation that results from the passage of light through a $\mathrm{Y}$ junction. The junction was composed of a single-mode waveguide with an index step of 0.0694 and a width of $2 \mu \mathrm{m}$. With the single bound eigenmode as an initial profile, the beam was propagated through a $Y$ junction defined by the union of two waveguides of the type mentioned above with centers located at $\pm[1-\cos (\pi z / 40)](t / 2)$ (all distances are in micrometers). 4 The calculation was performed for several values of the final waveguide separation, governed by the parameter $t$. The propagation was continued until only the bound mode remained in each waveguide, and the amount of power in the mode was noted. This calculation was done by using the TBC and was then repeated by using the artificial absorber method with standard Dirichlet boundary conditions. The absorber was constructed by linearly ramping the absorption coefficient to an optimal value, and it was extremely thick (145 zones) so as to serve as an accurate standard of comparison. Only half of the problem was zoned with 512 zones and a symmetry $(\partial E / \partial x$ $=0$ ) condition at the left boundary. Figure 2 shows the fraction of power remaining in the waveguide for each method as a function of $t$. The two methods agree to within $1 \%$ over the range of separations considered.

For the third test problem, the radiation loss of a three-dimensional $\mathrm{Y}$ junction was computed by using as before the TBC and absorber methods. In this case, however, the loss rate as well as the total loss was examined. The Y junction studied is a union of single-mode waveguides with the same shape function as in the two-dimensional case. The rib waveguide geometry employed (a structure that has been previously analyzed ${ }^{4}$ ) is shown in Fig. 3 . The TBC calculation was performed on a $4 \mu \mathrm{m} \times 4 \mu \mathrm{m}$ problem region subdivided into 64 horizontal and 80 vertical zones (only half of the problem was zoned in the horizontal direction). For the absorption method calculations, the problem region was expanded by adding additional absorption zones to the bottom and right bound-

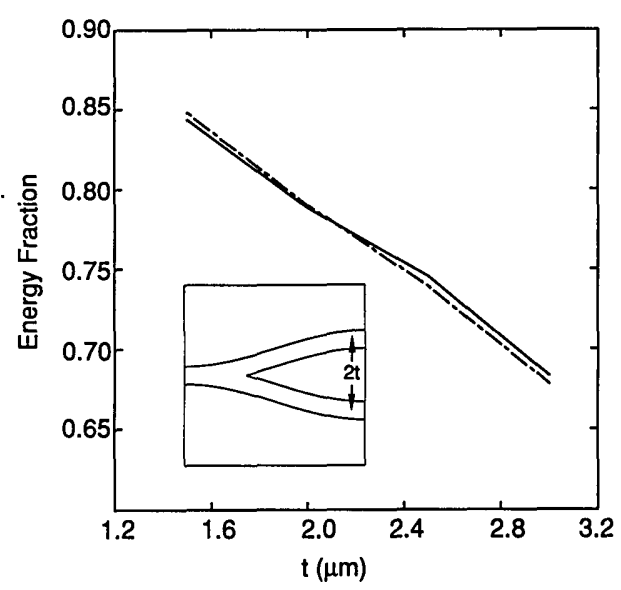

Fig. 2. Fraction of initial power remaining after the beam traverses a smooth two-dimensional $Y$ junction (wavelength $1.55 \mu \mathrm{m})$ and the radiative loss of all but the single bound mode. The curves are for the TBC method (solid curve) and the more conventional artificial absorber method (dashed curve) using 145 absorption zones. 


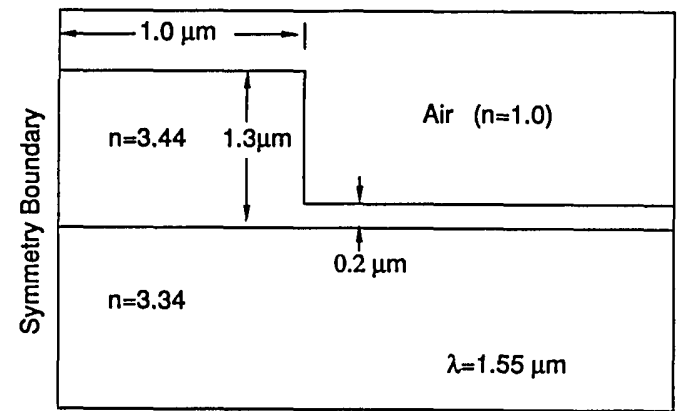

Fig. 3. Cross-section schematic of the rib waveguide used to construct the three-dimensional $Y$ junction for the third test. Only half of the device was zoned, with symmetry boundary conditions employed at the left boundary.

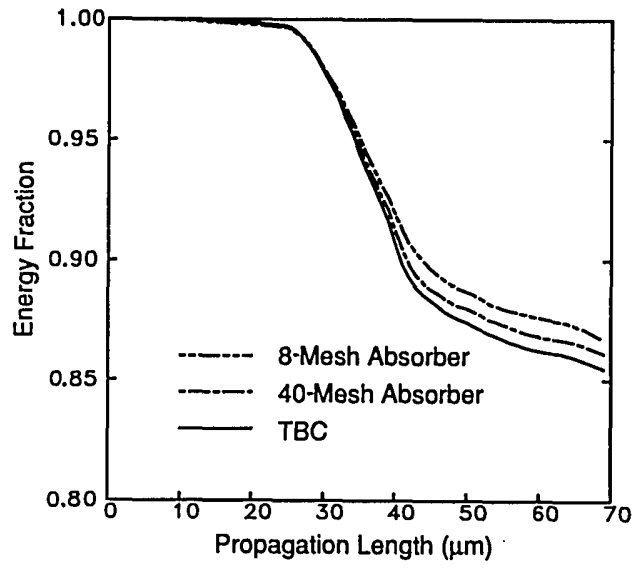

Fig. 4. Fraction of initial power remaining in a three-dimensional Y junction (wavelength $1.55 \mu \mathrm{m}$ ) versus propagation distance. The absorber method results seem to approach the TBC results as the absorption region thickness is increased. The absorption profile used was that given in Ref. 4.

aries with an absorption profile similar to that used in Ref. 4. However, only the energy within the original problem region was computed, in order to provide a consistent comparison between the two methods. In contrast to the second test case, the absorbers were of limited thickness (8 and 20 mesh points) owing to machine storage and run-time constraints. Figure 4 shows the fraction of the original energy remaining in the guides as a function of longitudinal position for both the TBC and absorber methods. Although the results for the 8-mesh absorber are in approximate agreement with previous calculations, ${ }^{4}$ the agreement is not exact because in Ref. 4 the energy in the absorbing region was included in the total. Figure 4 shows clearly that the amount of energy absorbed is still increasing as the number of absorption zones is increased to 20 and that these results appear to be approaching the TBC results. As a further check, the TBC calculation was run until a steady-state value for the remaining energy fraction was reached (a propagation distance of $7500 \mu \mathrm{m}$ ). At this point the energy fraction was 0.55 , in excellent agreement with the value 0.545 determined previously. The latter was calculated by computing the overlap integral of the solution at a distance of $40 \mu \mathrm{m}$ with the known bound eigenmode. ${ }^{6}$

The three test problems presented here show conclusively that the TBC performs as well as or better than the absorber method, without the need for tailoring to a specific problem or extra computational zones and without the ever-present uncertainty concerning absorber optimization. The method appears to provide effective reflection coefficients of approximately $10^{-5}$, although it should be kept in mind that this figure will depend somewhat on the angle of incidence and will worsen as grazing incidence is approached. Finally, the last two test problems illustrate the effectiveness of the TBC in treating radiation scattered to the boundaries through a wide range of angles. This method should thus see significant use in future beampropagation calculations and is therefore expected to affect strongly the design of complex optoelectronic structures.

This research was supported by the U.S. Department of Energy under contract DE-AC04-76DP00789.

\section{References}

1. A. Neyer, W. Mevenkamp, L. Thylen, and B. Lagerstrom, IEEE J. Lightwave Technol. LT-3, 635 (1985).

2. P. Kaczmarski and P. E. Lagasse, Electron. Lett. 24, 675 (1988).

3. J. Saijonmaa and D. Yevick, J. Opt. Soc. Am. 73, 1785 (1983).

4. D. Yevick and B. Hermansson, IEEE J. Quantum Electron. 25, 221 (1989).

5. R. Pregla and J. Gerdes, in Digest of Meeting on Integrated Photonics Research (Optical Society of America, Washington, D.C., 1990), paper MG3.

6. D. Yevick and B. Hermansson, IEEE J. Quantum Electron. 26, 109 (1990). 